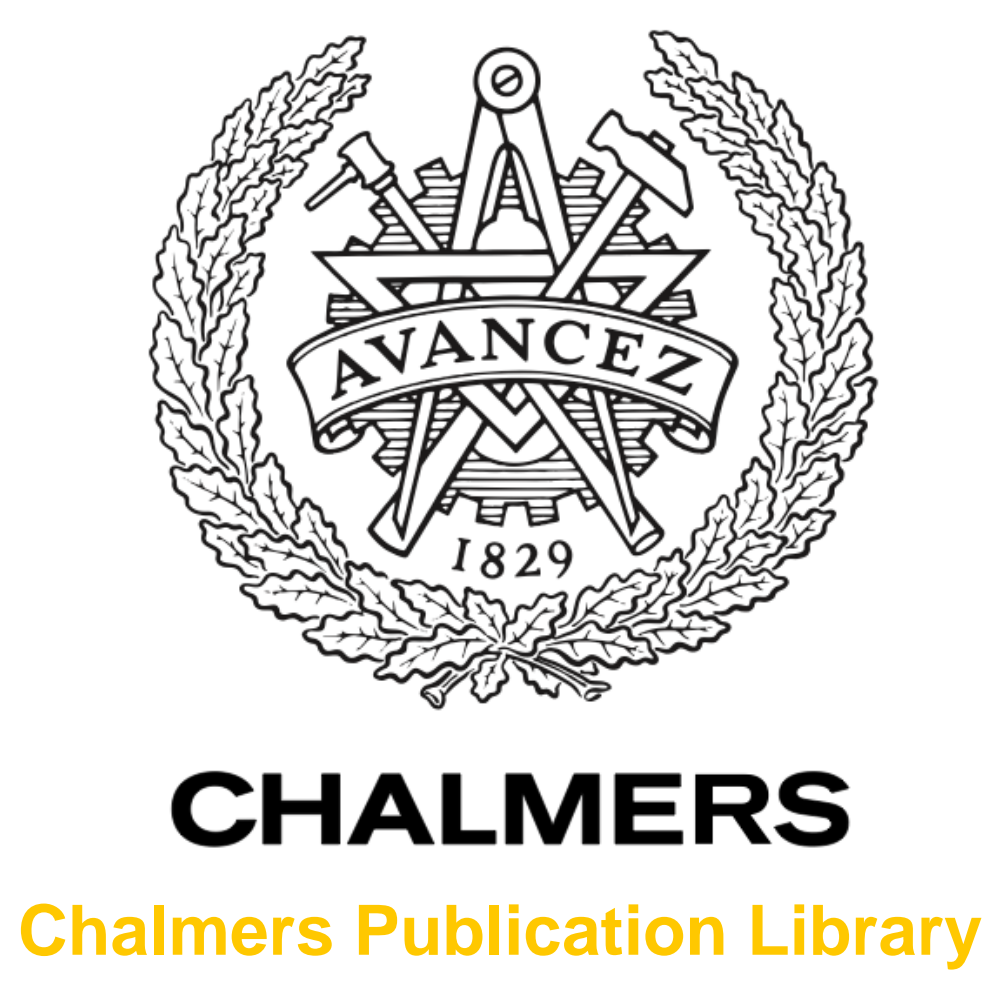

Towards an engineering model for curve squeal

This document has been downloaded from Chalmers Publication Library (CPL). It is the author's version of a work that was accepted for publication in:

Noise and Vibration Mitigation for Rail Transportation Systems. Proceedings of the 11th International Workshop on Railway Noise, 9-13 September 2013, Uddevalla, Sweden (ISSN: 1612-2909)

Citation for the published paper:

Zenzerovic, I. ; Pieringer, A. ; Kropp, W. (2015) "Towards an engineering model for curve squeal". Noise and Vibration Mitigation for Rail Transportation Systems. Proceedings of the 11th International Workshop on Railway Noise, 9-13 September 2013, Uddevalla, Sweden, vol. 126 pp. 433-440.

http://dx.doi.org/10.1007/978-3-662-44832-8_52

Downloaded from: http://publications.lib.chalmers.se/publication/188825

Notice: Changes introduced as a result of publishing processes such as copy-editing and formatting may not be reflected in this document. For a definitive version of this work, please refer to the published source. Please note that access to the published version might require a subscription.

Chalmers Publication Library (CPL) offers the possibility of retrieving research publications produced at Chalmers University of Technology. It covers all types of publications: articles, dissertations, licentiate theses, masters theses, conference papers, reports etc. Since 2006 it is the official tool for Chalmers official publication statistics. To ensure that Chalmers research results are disseminated as widely as possible, an Open Access Policy has been adopted.

The CPL service is administrated and maintained by Chalmers Library. 


\title{
Towards an Engineering Model for Curve Squeal
}

\author{
I. Zenzerovic, A. Pieringer and W. Kropp \\ Division of Applied Acoustics / CHARMEC, Chalmers University of Technology \\ SE - 41296 Göteborg, Sweden \\ Tel: +46 (0)31 772 2213, Fax: +46 (0)31 772 2212, E-mail: ivan.zenzerovic@chalmers.se
}

\section{Summary}

Curve squeal is a strong tonal noise that may arise when a railway vehicle negotiates a curve. The wheel/rail contact model is the central part of prediction models, describing the frictional instability occurring in the contact during squeal. A previously developed time-domain squeal model considers the wheel and rail dynamics, and the wheel/rail contact is solved using Kalker's nonlinear transient CONTACT algorithm with Coulomb friction. In this paper, contact models with different degree of simplification are compared to CONTACT within the previously developed squeal model in order to determine a suitable contact algorithm for an engineering curve squeal model. Kalker's steady-state FASTSIM is evaluated, and, without further modification, shows unsatisfying results. An alternative transient single-point contact algorithm named SPOINT is formulated with the friction model derived from CONTACT. Compared to the original model results, the SPOINT implementation results are promising and similar to results from CONTACT.

\section{Introduction}

Squeal noise is a strong tonal noise that may occur when a railway vehicle negotiates a relatively tight curve $(\mathrm{R}<200 \mathrm{~m}[1])$. The curve radius at which squeal is expected to occur depends on the vehicle bogie wheelbase. The wheelbase and curve radius define the angle of attack, which is considered the main kinematic parameter as it defines the amount of lateral creepage that occurs between wheel and rail [2].

The wheel and rail excitation force originates from the frictional instability occurring in the wheel/rail contact. This instability is caused by the slip velocity dependent falling friction characteristic of the wheel/rail contact and/or the vertical/lateral dynamic coupling of the wheel and rail (modes coupling) [3]. It is 
still not clear to what extent each of these factors contributes to the development of squeal, but cases where squeal is obtained either from simulations or experiments is reported for each cause (c.f. [3, 4, 5]) separately. Additionally, it is not yet fully understood which parameters affect the occurrence of curve squeal.

It is clear that the main part of any squeal model is the wheel/rail contact model. From the many available rolling contact models, Kalker's nonlinear transient CONTACT [6] and his linear steady-state FASTSIM [6, 13] algorithms are widely used. The recent time-domain squeal model developed by Pieringer [3], where CONTACT with Coulomb friction is used and the wheel and rail dynamics are included by means of Green's functions, is one of the most detailed models able to simulate curve squeal. However, CONTACT is computationally expensive and not viable in everyday engineering practice. Other time-domain squeal models, mainly using simplified contact algorithms, were developed e.g. by Fingberg [7], Périard [8], Huang et al. [9] and Heckl [10]. However, in those models falling friction curves are used, and the influence of different causes of curve squeal cannot be identified, nor can be the influence of different rolling contact algorithms.

The intention of this paper is to investigate, within Pieringer's squeal model [3], the suitability of different simplified rolling contact algorithms for use in a computationally efficient engineering model for curve squeal. At this point all contact algorithms apply the Coulomb friction model, or a model derived from it.

\section{Wheel and rail dynamics}

The squeal model [3] consists of three main submodels: wheel dynamics, rail dynamics, and the contact model that couples the wheel and rail. In [3], Pieringer includes the wheel and rail dynamics by means of Green's functions $g_{i j}$ obtained from the wheel and rail receptances using the inverse Fourier transform. The wheel and rail receptances are obtained from discrete models by modal superposition.

\subsection{Wheel model}

The wheel model is a finite element model based on axi-symmetric elements [3]. The modeled wheel is a $780 \mathrm{~mm} \mathrm{C20} \mathrm{metro} \mathrm{steel} \mathrm{wheel} \mathrm{with} \mathrm{a} \mathrm{Young's} \mathrm{modulus}$ $E=207 \mathrm{GPa}$, Poisson ratio $=0.3$ and density $=7860 \mathrm{~kg} / \mathrm{m}^{3}$. Only the lateral, vertical and vertical/lateral coupling dynamics are included in the model. The vertical/lateral coupling arises due to the asymmetry of the wheel cross section.

\subsection{Rail model}

The rail is described with a waveguide finite element model [3]. A continuously supported BV50 type rail is considered with the same material parameters as the wheel. The rail pad is also considered with the parameters: Young's modulus $E_{P}=4.8 \mathrm{MPa}$, Poisson ratio ${ }_{P}=0.45$ and density ${ }_{P}=10 \mathrm{~kg} / \mathrm{m}^{3}$. 
The main difference between the wheel and rail Green's functions is that the rail's functions are moving Green's functions. This means that the rail Green's functions describe the response of the rail when the excitation point is traveling along the rail [3].

\subsection{Dynamics response and convolution}

The dynamic response of the wheel and rail is obtained by convoluting the contact forces and the Green's function of the respective body. A discrete version of the Green's functions and convolution is used in the algorithms (c.f. [3]):

$$
{ }_{i}\left(t_{k}\right)={ }_{t=0}^{t_{k}}{ }_{j=2}^{3} F_{j}(t) g_{i j}\left(t_{k} \quad t\right)={ }_{j=2}^{3} F_{j}\left(t_{k}\right) g_{i j}(0)+{ }_{t=0}^{t_{k} 13} F_{j=2}(t) g_{i j}\left(t_{k} \quad t\right),
$$

where $g_{i j}(0)$ is the first value of the Green's function, which gives the local instantaneous deformation of the body due to the excitation force in the current time step. The $g_{i j}(0)$ term can be treated as the dynamic flexibility coefficient, which accounts for the dynamic effects of the system [11]. The second sum on the right-hand side of equation (2.1) gives the dynamic response due to forces acting in previous time steps. The indices $i, j=1,2,3$ refer to the longitudinal, lateral and vertical directions of the contact coordinate system.

\section{Contact models}

Every rolling contact model consists of two main submodels: a normal and a tangential contact model. The contact model couples the wheel and rail dynamics, and the tangential contact model is crucial for simulating the frictional instability occurring in curve squeal.

\subsection{CONTACT}

Kalker's CONTACT [6] consists of the non-Hertzian normal contact algorithm NORM and the transient tangential contact algorithm TANG. The first values of the wheel and rail Green's functions are neglected, which significantly simplifies the algorithm. Both NORM and TANG algorithms are based on the elastic half-space assumption and the Boussinesq-Cerruti equations. While NORM determines the elements in contact, TANG determines the stick and slip regions of the contact area. The wheel and rail dynamic contributions are included in the per-element rigid shift:

$$
W_{l 1}=\left(\begin{array}{cc}
x & y_{l}
\end{array}\right) x, \quad W_{l 2}=\left(\begin{array}{l}
y \\
y
\end{array}\right) x+\left(\begin{array}{cc}
R & R t_{i} 1 \\
2 & 2
\end{array}\right)\left(\begin{array}{cc}
W & W, t_{i} 1 \\
2 & 2
\end{array}\right)
$$

where $x, y$ and are the longitudinal, lateral and spin creepages, $x$ is the discretization element length, and $\left(x_{l}, y_{l}\right)$ the element center coordinates in the contact coordinate system. The dynamics of the wheel and rail are included by means of the current time step responses ${ }_{2}^{W}$ and ${ }_{2}^{R}$, and the previous time step responses $2_{2}^{W, t_{i} 1}$ and ${ }_{2}^{R t_{i} 1}$. 
Both NORM and TANG are iterative active set algorithms [6]. The non-linear system of equations in TANG is solved using the Newton-Raphson method.

\subsection{FASTSIM}

Kalker's steady-state FASTSIM $[6,13]$ is based on the simplified theory where the deformation at a point of the contact area depends only on the load at that point. The normal contact problem is solved using the Hertz contact theory.

The effective, per-element, rigid slip is modified to include the dynamic response of the wheel and rail:

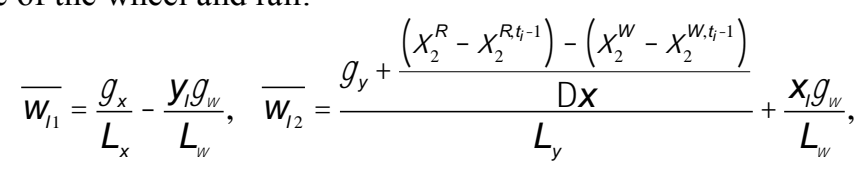

where $L_{x}, L_{y}$ and $L$ are the flexibility parameters computed from the semi-axes ratio $a / b$ of the contact ellipse and the creepage coefficients $C_{i j}$. The creepage coefficients are tabulated in [6].

3.3

SPOINT

The single-point normal and tangential contact problems are solved simultaneously. The wheel and rail dynamics terms, that contain the first values of the Green's functions, are included in the contact point gross slip velocity:

$$
\begin{aligned}
& S=\left(x+\frac{u_{1} u_{1}^{t_{1}}}{x}\right) V
\end{aligned}
$$

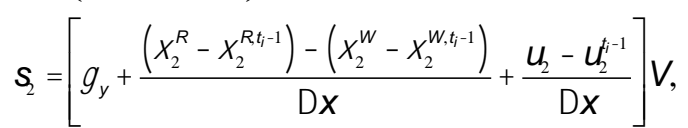

where $u_{1}$ and $u_{2}$ are the contact deformations in the current time step, $u_{1}^{t_{1} 1}$ and $u_{2}^{t_{1} 1}$ the contact deformations in the previous time step, and $V$ the rolling velocity. The system of equations defining the single-point contact is:

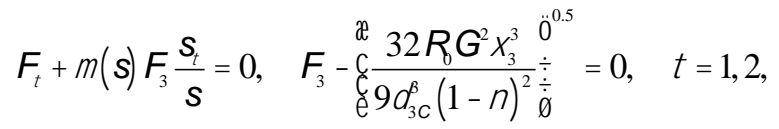

where $s=\sqrt{s_{1}^{2}+s_{2}^{2}}$ is the absolute slip velocity, $3 c$ a constant depending on the ratio $a / b, R_{0}$ the effective radius of curvature, and ${ }_{3}$ is the combined wheel/rail vertical dynamics contribution. The contact deformation is computed as $u=K F$, with $K$ being the contact compliance obtained from the vertical contact stiffness linearized around the vertical preload. According to [12], the lateral contact stiffness is $20 \%$ higher than the vertical stiffness. The contact plane compliance is then $K=K_{3} / 1.2$ with $K_{3}$ being the vertical contact compliance.

The resulting system of equations is solved using the Newton-Raphson method. In its current formulation, SPOINT is unable to account for spin creepage. 


\subsubsection{Friction model}

When using a single-point contact, it is essential that the friction model is formulated in a stringent way in relation to the "multi-point" contact where Coulomb friction is applied. Therefore, for each value of the friction coefficient, CONTACT results are obtained for slowly linearly varying lateral creepage $\left(y_{y}=\lim _{y} t / t_{\text {end }}, \quad \lim _{y}=0.05, t_{\text {end }}=2 s\right)$ and no wheel $/$ rail dynamics included. Due to the slow change of creepage, a steady-state solution is assumed for each time step. The friction curve (traction coefficient) is determined as $s={ }_{y} V, \quad(s)=F_{2} / F_{3}$. In that way the friction model for SPOINT is obtained as shown in Fig.1.

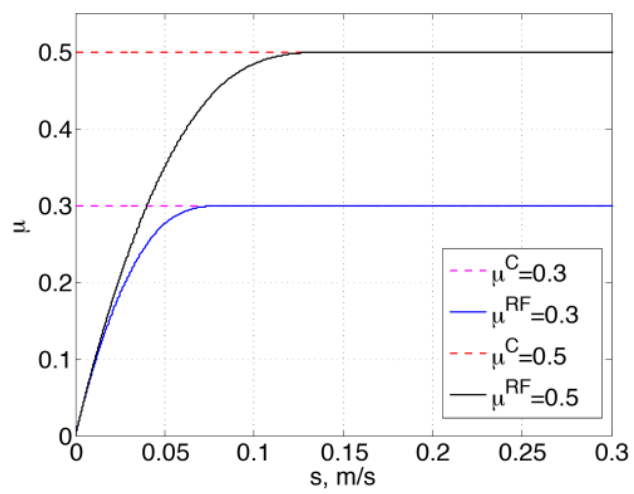

Fig. 1. Comparison of the friction model used in SPOINT and Coulomb friction for two values of the friction coefficient 0.3 and 0.5 . RF - regularized friction; $\mathrm{C}-$ Coulomb.

The friction model has the form of a regularized friction (RF). With increasing Coulomb friction in CONTACT the transition region of the RF (i.e. the region before the curve converges to a constant value) is extended.

The results underline the importance to distinguish between local and global friction models. The local friction model applies to a single contact element (or particle in contact), while the global friction model applies to the complete contact. The discretization of the contact and the elastic half-space in CONTACT, as a third body between the wheel and rail, soften the friction curve for small slip velocities. By using the CONTACT steady-state solution as input for the friction model in SPOINT, this effect is accounted for in the simplified approach. The results also highlight the question how an appropriate friction model for real cases should be formulated at all.

\section{$4 \quad$ Results}

The occurrence of stick-slip oscillations, signifying curve squeal, can be determined from the time histories of the lateral contact force obtained from simulations with the squeal model. Simulations were performed for a vehicle 
velocity of $V=50 \mathrm{~km} / \mathrm{h}$ and the time step length follows from the kinematic relationship $t=x / V(x=0.5 \mathrm{~mm})$. The amplitude of the lateral force oscillations is quantified with the lateral force level $L_{F 2}$ based on the root mean square (RMS) value of the lateral force as:

$$
L_{F_{2}}=20 \log F_{2, r m s}, \quad F_{2, r m s}=\sqrt{\frac{1}{n}{ }_{1}^{1+n}\left(F_{2}() \overline{F_{2}}\right)^{2} \mathrm{~d}},
$$

where $n=1000$ is the number of time samples considered, $\overline{F_{2}}$ the mean value of the force in the considered time interval, and $\alpha$ is the discrete time step number.

\subsection{FASTSIM}

The steady-state FASTSIM, in its original formulation, seems not to be appropriate to simulate curve squeal. Results were not physical in all analyzed cases. Obviously, FASTSIM is not suitable for solving the rolling contact problem when the wheel and rail dynamics are included directly in the rigid slip, c.f. equation (3.2). However, the application of a transient algorithm based on FASTSIM (see e.g. [14]) could solve this problem. The inclusion of the contact area deformation history or previous time step tractions distribution enhances the simulation behavior and physical results can be obtained. However, these additional terms alter the original FASTSIM algorithm changing its properties and further investigations might be needed.

\subsection{SPOINT}

In Fig. 2 the CONTACT and SPOINT dynamic simulation results are shown in terms of the lateral force levels. Simulations were performed for different combinations of friction coefficient and lateral creepage values. Except for high friction values, very good agreement is found between CONTACT and SPOINT results.
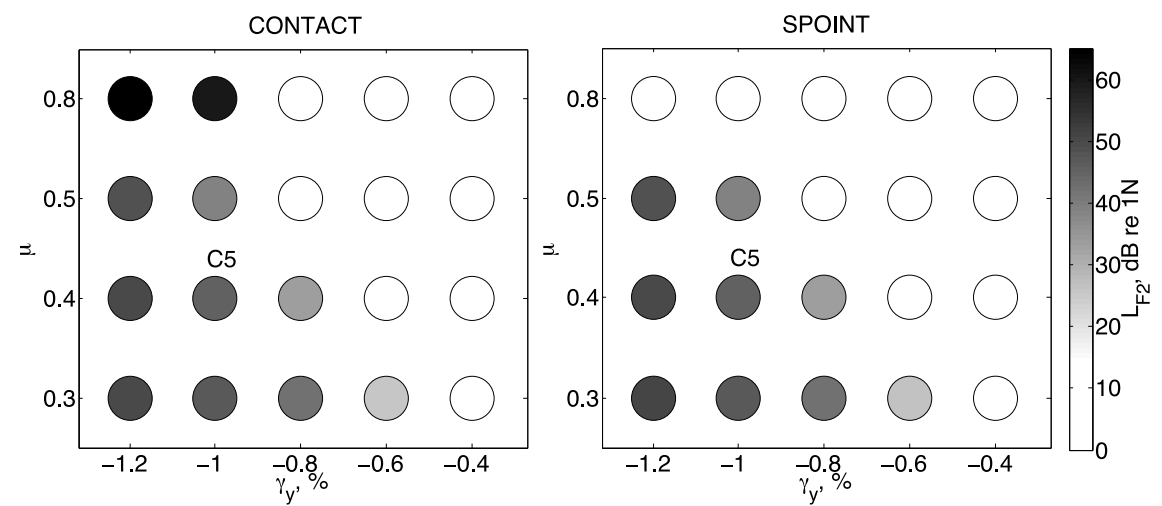

Fig. 2. CONTACT and SPOINT dynamic simulations results presented in terms of RMS values. The case 5 is denoted with $\mathrm{C} 5$. 

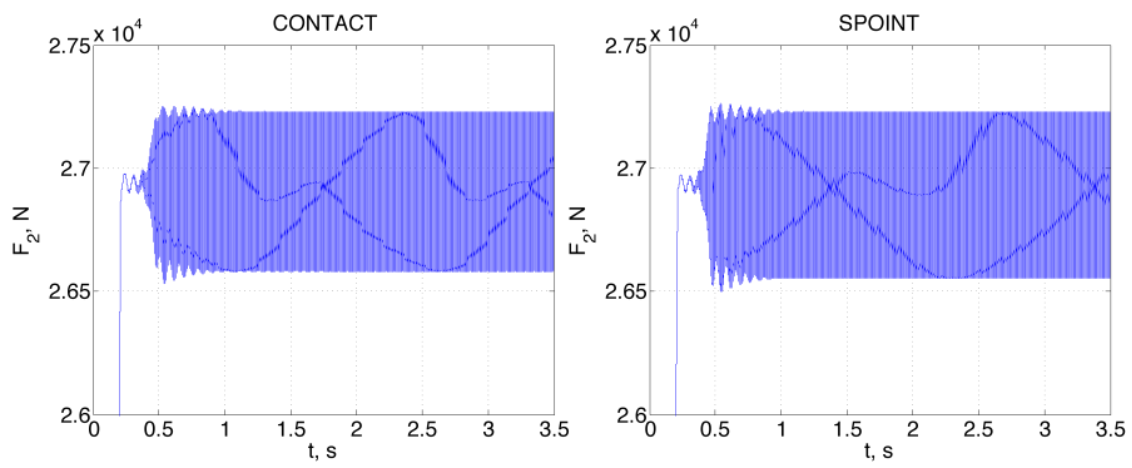

Fig. 3. Lateral force time histories of case $5(y=0.01, \quad=0.4)$ obtained with CONTACT and SPOINT.

In Fig. 3 and Fig. 4, the time histories of a single simulation and the details of the occurring stick-slip lateral force oscillations are shown respectively. Very good agreement is found in the stick-slip oscillation details. Deviations between CONTACT and SPOINT results can be due to spatial and time discretization and the uncertainties regarding the contact longitudinal and lateral stiffnesses, which are a required input parameter in SPOINT.
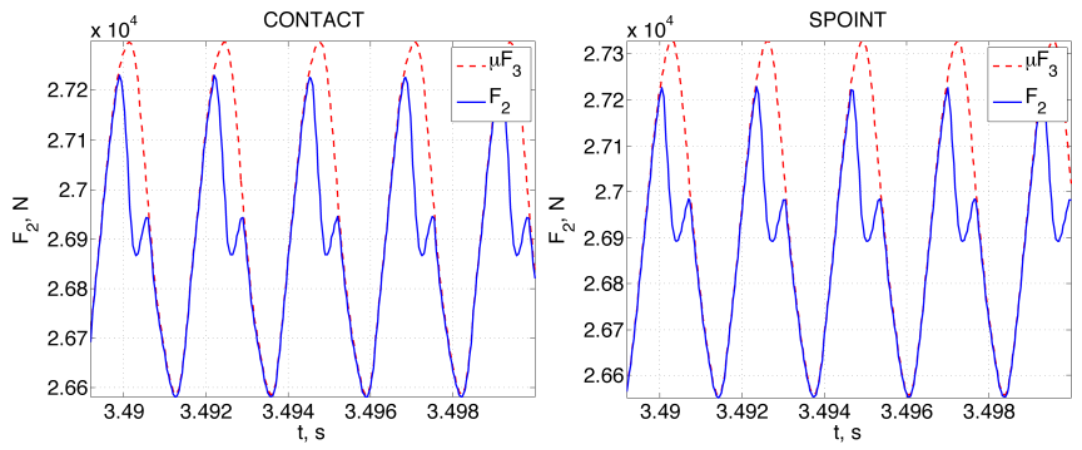

Fig. 4. Details of the lateral contact force oscillations due to stick-slip occurring in the contact for case 5 .

\section{Conclusion}

Two alternative contact formulations were compared to the results of CONTACT, as used in [3], with respect to simulations of squealing. The contact deformation history, neglected in steady state FASTSIM, was shown to be crucial to obtain reasonable results from FASTSIM. The single-point contact showed very good agreement with CONTACT. This however demands that the global friction model is derived from CONTACT. However, in practice it might be easier to obtain such global friction models (e.g. from traction curves) than the local friction model for CONTACT. 


\section{Acknowledgements}

The presented work has been performed within the project "Abatement of Curve Squeal Noise from Trains" (VB11) and is part of the activities in the Centre of Excellence CHARMEC (CHAlmers Railway MEChanics).

\section{References}

[1] Thompson, D.J.: Railway Noise and Vibration: Mechanisms, Modelling and Means of Control, Elsevier, Oxford, UK (2009).

[2] Vincent, N. et al: Curve squeal of urban rolling stock - Part 1: State of art and field measurements, Journal of Sound and Vibration. 293, 691-700 (2006).

[3] Pieringer, A.: Time-domain modelling of high-frequency wheel/rail interaction, PhD Thesis, Chalmers University of Technology (2011).

[4] Rudd, M.J.: Wheel/Rail Noise - Part II: Wheel Squeal, Journal of Sound and Vibration. 46(3), 381-394 (1976).

[5] Koch, J.R. Vincent, N. Chollet, H. Chiello, O.: Curve squeal of urban rolling stock - Part 2: Parametric study on a $1 / 4$ scale test rig, Journal of Sound and Vibration. 293, 701-709 (2006).

[6] Kalker, J.J.: Three-Dimensional Elastic Bodies in Rolling Contact, Kluwer Academic Publishers, Dordrecht, The Netherlands (1990).

[7] Fingberg, U.: A model of wheel-rail squealing noise, Journal of Sound and Vibration. 143(3), 365-377 (1990).

[8] Périard, F.J.: Wheel-Rail Noise Generation: Curve Squealing by Trams, $\mathrm{PhD}$ Thesis, Technische Universiteit Delft (1998).

[9] Huang, Z.Y., Thompson, D.J., Jones, C.J.C.: Squeal prediction for a bogied vehicle in a curve, Notes on Numerical Fluid Mechanics and Multidisciplinary Design, 99, 313-319 (2008).

[10] Heckl, M.A. Abrahams, I.D.: Curve squeal of train wheels, Part 1: Mathematical model for its generation, Journal of Sound and Vibration. 229(3), 669-693 (2000).

[11] Andersson, P.: Modelling Interfacial Details in Tyre/Road Contact Adhesion Forces and Non-Linear Contact Stiffness, PhD Thesis, Chalmers University of Technology (2005).

[12] Wu, T.X. Thompson, D.J.: Wheel/Rail Non-Linear Interaction with Coupling between Vertical and Lateral Directions, Vehicle System Dynamics, 41(1), 27-49 (2004).

[13] Kalker, J.J.: A Fast Algorithm for the Simplified Theory of Rolling Contact, Vehicle System Dynamics. 11, 1-13 (1982).

[14] Guiral, A. Alonso, A. Baeza, L. Giménez, J.G.: Non-steady state modelling of wheel-rail contact problem, Vehicle System Dynamics, 51(1), 91-108 (2013). 